\title{
A DGTD Scheme for Modeling the Radiated Emission From DUTs in Shielding Enclosures Using Near Electric Field Only
}

\author{
Ping Li, Member, IEEE, Yifei Shi, Member, IEEE, Li Jun Jiang, Senior Member, IEEE, \\ and Hakan Bağc1, Senior Member, IEEE
}

\begin{abstract}
To meet the electromagnetic interference regulation, the radiated emission from device under test such as electronic devices must be carefully manipulated and accurately characterized. Instead of resorting to the direct far-field measurement, in this paper, a novel approach is proposed to model the radiated emission from electronic devices placed in shielding enclosures by using the near electric field only. Based on the Schelkkunoff's equivalence principle and Raleigh-Carson reciprocity theorem, only the tangential components of the electric field over the ventilation slots and apertures of the shielding enclosure are sufficient to obtain the radiated emissions outside the shielding box if the inside of the shielding enclosure was filled with perfectly electric conductor (PEC). In order to efficiently model wideband emission, the time-domain sampling scheme is employed. Due to the lack of analytical Green's function for arbitrary PEC boxes, the radiated emission must be obtained via the full-wave numerical methods by considering the total radiated emission as the superposition between the direct radiation from the equivalent magnetic currents in free space and the scattered field generated by the PEC shielding box. In this study, the state-of-the-art discontinuous Galerkin time-domain (DGTD) method is utilized, which has the flexibility to model irregular geometries, keep high-order accuracy, and more importantly involves only local operations. For open-region problems, a hybridized DGTD and time-domain boundary integration method applied to rigorously truncate the computational domain. To validate the proposed approach, several representative examples are presented and compared with both analytical and numerical results.
\end{abstract}

Index Terms-Boundary integration (BI) method, discontinuous Galerkin time-domain (DGTD) method, electronic systems in shielding enclosure, equivalence principle, radiated emission, Raleigh-Carson reciprocity theorem.

\section{INTRODUCTION}

$\mathbf{T}$ ODAY'S electronic systems consisting of highly populated RF modules, digital dies, and printed circuit boards (PCB) become an efficient radiator as frequencies increase [1][3]. To meet the electromagnetic interference regulation, the

Manuscript received June 1, 2015; revised September 16, 2015; accepted November 27, 2015. This work is supported in part by the National Science Foundation of China under Grant 61234001.

P. Li, Y. Shi, and H. Bağcı are with the Division of Computer, Electrical, and Mathematical Sciences and Engineering and the Center for Uncertainty Quantification in Computational Science and Engineering, King Abdullah University of Science and Technology, Thuwal 23955-6900, Saudi Arabia (e-mail: ping.li@kaust.edu.sa; yifei.shi@kaust.edu.sa; hakan.bagci@kaust.edu.sa).

L. J. Jiang is with the Department of Electrical and Electronic Engineering, The University of Hong Kong, Hong Kong (e-mail: jianglj@hku.hk).

Color versions of one or more of the figures in this paper are available online at http://ieeexplore.ieee.org.

Digital Object Identifier 10.1109/TEMC.2016.2515363 radiated emissions from noise sources such as victim traces due to crosstalk, discontinuities of return currents happening at conducting trace-vias transitions, and propagating of the switching noise along the power/ground plate guide [4] must be controlled below the required level. Admittedly, the conventional full-wave numerical approaches such as finite-difference timedomain method [5]-[7], finite-element method (FEM) [8], and method of moments [9] are accurate by directly solving the Maxwell's equations. However, the computational cost is too expensive since the multiscale geometries result in poorly conditioned matrix equations with millions of unknowns.

As an alternative, the straightforward far-field (FF) measurement conducted in the open area test site (OATS) or semianechoic chambers is also a widely referred technique in electromagnetic compatibility society [10]. Usually, the maximum radiation at 3 or $10 \mathrm{~m}$ away from the electronic system is of interest [10], [11]. Unfortunately, this method is time consuming, prohibitively expensive, and requires electromagnetic (EM) cleaning environments for OATS. Therefore, other remedies must be developed.

Rigorously, the aforeproposed approaches can be categorized into two groups. For the first group, the mode-expansion-based technique is involved. In [12], the electric field is first measured over a spherical surface enclosing the antenna, and then, spherical wave modes are used to expand the measured fields. Based on the orthogonal property of the spherical wave modes, the expansion coefficients can be conveniently calculated. Similarly, the electric field is sampled over a cylindrical surface, and then, expanded by cylindrical wave modes [13]. Later, the planarly scanned near-electric field are approximated by a number of plane-waves in the spectral domain [14]. Via Fourier transform, the FF over a certain rectangular plane in front of the antenna can be predicted. Instead of direct expanding the field via mode functions, in [15], the Dyadic Green's function in the presence of perfectly electrical conducting sphere is expanded by spherical wave modes. With this Green's function, the field outside the measurement spherical surface can be straightforwardly obtained based on the Huygens' principle. The main advantage of this approach is that the Green's function is valid at any frequency but only requires a single calculation. Thus, it is more time efficient.

As for the second approach, the original radiators are replaced by a set of equivalent current sources that are able to reproduce the original emission. The equivalent source can be either electric, magnetic currents, or Hertzian dipoles. In [16], the 
radiated near electric field of the antenna is sampled over a rectangular plane in front of the antenna. To generate the same radiation, the antenna is substituted by a surface magnetic current over an infinitely large PEC plane. Via point matching technique, the unknown magnetic current is inversely determined based on conjugate gradient method. In [17], this approach is directly extended to the equivalent electric current source situation. The main deficiency of these two methods is that only the radiation in the forward half-space can be predicted. To overcome this problem, [18]-[22] proposed to construct the equivalent current source over the surface of the radiator and simultaneously the near-field is sampled over a surface enclosing the radiator. To guarantee the continuous property of the equivalent current, the Rao-Wilton-Glisson [23] basis function is used to expand the unknown current. By point-matching method, the expansion coefficient can be calculated through iterative methods. Other alternative sources such as Hertzian dipoles are also deployed to reproduce the radiated emissions from PCBs and ICs in [25]. In [24], the parameters of the equivalent dipole sources are optimized by genetic algorithm. For the equivalent source approach, the inverse process is involved which could be prohibitive cost due to the ill-conditioned matrix equations. Furthermore, the established current source could be nonunique due to the existence of nonradiating sources.

In this paper, a novel approach based on the near electric field only is proposed to characterize the radiated emission from electronic systems placed in shielding enclosures. Usually, the surface of the shielding enclosure can be assumed as PEC, thus, only the tangential components of electric field over the ventilation slots and apertures plus the tangential components of the magnetic field over the whole surface of the enclosure are needed according to the Huygens' principle. By further referring to the Love's equivalence principle [26], the inside of the Huygens' surface can be filled with arbitrary material without affecting the outgoing radiation. Thus, we fill the inside of the shielding enclosure with PEC. As a result, only the contribution from the equivalent magnetic current (derived from the near electric field: $\mathbf{M}_{s}=-\hat{\mathbf{n}} \times \mathbf{E}$ ) over the ventilation slots and apertures are needed since the electric current (derived from the near magnetic field: $\mathbf{J}_{s}=\hat{\mathbf{n}} \times \mathbf{H}$ ) over a PEC surface is nonradiative in line with the Raleigh-Carson reciprocity theorem. In this way, the number of NF sampling is drastically reduced. On the other hand, a powerful approach should be capable of efficiently predicting the radiated emission over a very broad frequency band. Thus, time-domain strategy is more preferable compared with frequency-domain method since only single NF-FF transformation is sufficient. To reach this aim, the near electric field is recorded in the time domain. In order to obtain the radiation from the equivalent magnetic current source over the PEC-filled shielding enclosure, the original problem is equivalently transformed to a scattering problem by considering the total radiation as the superposition between the direct radiation from the magnetic current source in free face and the scattered field by the PEC shielding box. In this study, the discontinuous Galerkin time-domain (DGTD) method [27]-[29] is utilized to solve this scattering issue. Compared with the approach in [30], the near electric field is only required over the slots and apertures over
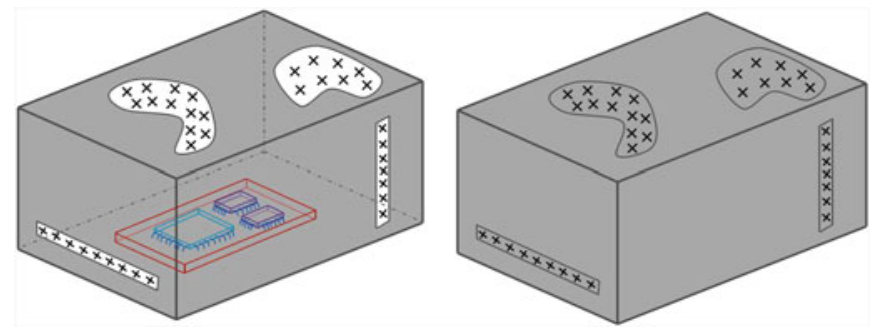

Fig. 1. Left: The electronic system placed into a shielding enclosure, and the tangential components of the electric field are temporally sampled over the ventilation slots and apertures by an electric dipole. Right: The equivalence of the original problem by filling the inside of the shielding enclosure with PEC. The equivalent magnetic current $\mathbf{M}_{s}$ is able to reproduce the original radiated emission by the superposition of the direct radiation from $\mathbf{M}_{s}=-\hat{\mathbf{n}} \times \mathbf{E}_{\mathrm{m} \text { eas }}$ in free space and the scattered field from the PEC filled shielding box.

the shielding enclosure while it requires the magnetic field over the whole surface of the shielding enclosure in [30] since the tangential components of the magnetic field is nonzero over the PEC surface.

As the combination of finite volume method [31] and FEM [32], all spatial operations of DGTD are local due to the use of numerical flux for information exchange between neighboring elements. Thus, the resultant mass-matrices are blockdiagonal and can be inverted before starting time-marching scheme. Consequently, a compact and efficient solver when combined with an explicit time marching scheme is reached. Due to the existence of the equivalent magnetic surface current, the discontinuity of the tangential electric field must be considered when deriving the numerical flux expressions based on the Rankine-Hugoniot Jump Relations along the characteristics of hyperbolic systems. For this open-region scattering problem, the DGTD is integrated with the time-domain boundary integral (TDBI) algorithm to truncate the computational domain by evaluating the field values required for the incoming numerical flux calculation over the truncation boundary [33]. It is mathematically exact, and the truncation boundary can be conformal to the surface of the shielding enclosure, thus making the computational region as small as possible.

The remainder of this paper organized as follows. In Section II, the basic EM theories and DGTD formulations are detailed, including the clarification of equivalence principle-based method, the derivation of the numerical flux and the formulation of matrix equations. Section III will benchmark various canonical examples to verify the accuracy and robustness of the algorithm. Finally, conclusions and discussions are presented at the end of the paper.

\section{THEORY AND MATHEMATICAL FORMULATION}

For electronic systems in shielding enclosures as shown in Fig. 1, both the electric and magnetic fields over the surface of the shielding enclosure are needed according to the Huygens' principle, which is time consuming and also very difficult. Therefore, it is more preferable if only electric or magnetic field was required. According to Love's equivalence principle and Raleigh-Carson reciprocity theorem [26], [34], the equivalent electric currents $\mathbf{J}_{s}\left(\mathbf{r}^{\prime}, t\right)=\hat{\mathbf{n}} \times \mathbf{H}\left(\mathbf{r}^{\prime}, t\right)$ is nonradiative if the 
inside of the shielding enclosure is filled up with PEC. Consequently, only the tangential components of the electric field (or the equivalent magnetic currents $\mathbf{M}_{s}\left(\mathbf{r}^{\prime}, t\right)=\mathbf{E}\left(\mathbf{r}^{\prime}, t\right) \times \hat{\mathbf{n}}$ ) over the ventilation slots and apertures are required to predict the radiated emission outside the shielding enclosure based on the Schelkkunoff's equivalence principle.

However, the radiated fields cannot be analytically calculated since the DGF in the presence of arbitrary PEC box is not available [37]. However, the calculation of the numerical DGF is prohibitively expensive. In this study, the DGTD method is employed by considering the problem as a scattering issue with incident field generated from $\mathbf{M}_{s}\left(\mathbf{r}^{\prime}, t\right)$.

Due to the existence of the surface magnetic current $\mathbf{M}_{s}$, the following boundary condition over the ventilation slots and apertures must be satisfied

$$
\hat{\mathbf{n}} \times\left(\mathbf{E}^{+}-\mathbf{E}^{-}\right)=-\mathbf{M}_{s}
$$

with superscripts + and - denoting the two sides of the impedance surface and $\hat{\mathbf{n}}$ representing the unit normal vector pointing from - to + .

\section{A. Numerical Flux Derivation}

To incorporate the boundary condition in 1) into DGTD, the mathematical expression of the numerical flux should be reformulated. For an arbitrary mesh element $i$ (tetrahedrons are used in this study), the Rankine-Hugoniot Jump Relations along the three different characteristics [31] for the $f$ th face of element $i$ are defined as

1) Jump across the characteristic $x_{n}=-c^{i} t$

$$
\begin{aligned}
\frac{1}{\mu^{i}} \hat{\mathbf{n}}_{i, f} \times\left(\mathbf{E}_{f}^{*}-\mathbf{E}^{i}\right) & =-c^{i}\left(\mathbf{H}_{f}^{*}-\mathbf{H}^{i}\right) \\
\frac{1}{\epsilon^{i}} \hat{\mathbf{n}}_{i, f} \times\left(\mathbf{H}^{i}-\mathbf{H}_{f}^{*}\right) & =-c^{i}\left(\mathbf{E}_{f}^{*}-\mathbf{E}^{i}\right) .
\end{aligned}
$$

2) Jump across the characteristic $x_{n}=0$

$$
\begin{aligned}
\hat{\mathbf{n}}_{i, f} \times\left(\mathbf{E}_{f}^{* *}-\mathbf{E}_{f}^{*}\right) & =-\mathbf{M}_{s} \\
\hat{\mathbf{n}}_{i, f} \times\left(\mathbf{H}_{f}^{* *}-\mathbf{H}_{f}^{*}\right) & =0 .
\end{aligned}
$$

3) Jump across the characteristic $x_{n}=c_{f}^{j} t$

$$
\begin{aligned}
& \frac{1}{\mu_{f}^{j}} \hat{\mathbf{n}}_{i, f} \times\left(\mathbf{E}_{f}^{j}-\mathbf{E}_{f}^{* *}\right)=c_{f}^{j}\left(\mathbf{H}_{f}^{j}-\mathbf{H}_{f}^{* *}\right) \\
& \frac{1}{\epsilon_{f}^{j}} \hat{\mathbf{n}}_{i, f} \times\left(\mathbf{H}_{f}^{* *}-\mathbf{H}_{f}^{j}\right)=c_{f}^{j}\left(\mathbf{E}_{f}^{j}-\mathbf{E}_{f}^{* *}\right)
\end{aligned}
$$

where $1 \leq f \leq 4$ for tetrahedrons, $j$ denotes the neighboring element sharing the $f$ th face of element $i, \hat{\mathbf{n}}_{i, f}$ is the outward unit normal vector of face $f . c^{i}$ and $c_{f}^{j}$ represent the characteristic speed in element $i$ and its neighboring $j$, respectively. $\epsilon^{i / j}$ and $\mu^{i / j}$ are the permittivity and permeability, respectively. $\left(\mathbf{E}_{f}^{*}, \mathbf{H}_{f}^{*}\right)$ and $\left(\mathbf{E}_{f}^{* *}, \mathbf{H}_{f}^{* *}\right)$ are the intermediate states in element $i$ and $j$, respectively.
By combining (2), (4), (5), and (6), the upwind flux for the Ampere's law equation is formulated by

$$
\begin{aligned}
\hat{\mathbf{n}}_{i, f} \times \mathbf{H}_{f}^{*}= & \hat{\mathbf{n}}_{i, f} \times \frac{\left(Z^{i} \mathbf{H}^{i}+Z_{f}^{j} \mathbf{H}_{f}^{j}\right)+\hat{\mathbf{n}}_{i, f} \times\left(\mathbf{E}^{i}-\mathbf{E}_{f}^{j}\right)}{Z^{i}+Z_{f}^{j}} \\
& -\frac{\hat{\mathbf{n}}_{i, f} \times \mathbf{M}_{s}}{\left(Z^{i}+Z_{f}^{j}\right)} .
\end{aligned}
$$

Similarly, with (3), (4), (5), and (7), the upwind flux for the Maxwell-Faraday's law equation is given by

$$
\begin{aligned}
\hat{\mathbf{n}}_{i, f} \times \mathbf{E}_{f}^{*}= & \hat{\mathbf{n}}_{i, f} \times \frac{\left(Y^{i} \mathbf{E}^{i}+Y_{f}^{j} \mathbf{E}_{f}^{j}\right)+\hat{\mathbf{n}}_{i, f} \times\left(\mathbf{H}_{f}^{j}-\mathbf{H}^{i}\right)}{Y^{i}+Y_{f}^{j}} \\
& +\frac{Y_{f}^{j} \mathbf{M}_{s}}{2\left(Y^{i}+Y_{f}^{j}\right)}
\end{aligned}
$$

where $Z^{i}=\sqrt{\mu^{i} / \epsilon^{i}}$ and $Z_{f}^{j}=\sqrt{\mu_{f}^{j} / \epsilon_{f}^{j}}$ represent the characteristic impedance of element $i$ and the neighboring at the $f$ th face.

Since the surface magnetic current $\mathbf{M}_{s}$ is over the PEC, therefore the parameters of the neighboring element $j$ are: $Z_{f}^{j}=0$ and $Y_{f}^{j}=\infty$. For programming convenience, this can be equivalently achieved by letting $\mathbf{E}_{f}^{j}=-\mathbf{E}^{i}, \mathbf{H}_{f}^{j}=\mathbf{H}^{i}, Z_{f}^{j}=Z^{i}$ and $Y_{f}^{j}=Y^{i}[35]$.

\section{B. DGTD Formulation}

Let $\Omega$ denote the computation domain of interest, which is bound by surface $\partial \Omega$. With DGTD, the domain $\Omega$ is first split into $N$ nonoverlapping tetrahedrons $\Omega_{i}$ with boundary $\partial \Omega_{i}$. In element $i$, the electric field $\mathbf{E}$ and magnetic field $\mathbf{H}$ are approximated by vector basis functions $\Phi^{i}(\mathbf{r})$ and $\Psi^{i}(\mathbf{r})$ [29], [33]: $\mathbf{E}^{i}=\sum_{k=1}^{n_{e}^{i}} e_{k}^{i}(t) \boldsymbol{\Phi}_{k}^{i}(\mathbf{r})$ and $\mathbf{H}^{i}=\sum_{l=1}^{n_{h}^{i}} h_{k}^{i}(t) \boldsymbol{\Psi}_{l}^{i}(\mathbf{r})$, where $n_{e}^{i}$ and $n_{h}^{i}$ are the number of vector basis function [32] for $\mathbf{E}$ and $\mathbf{H}$ in $i$ th element, respectively; $e_{k}^{i}$ and $h_{k}^{i}$ are the unknown time-dependent coefficients.

By applying the DG testing over the two first-order timederivative Maxwell's curl equation, we can obtain

$$
\begin{aligned}
& \int_{\Omega_{i}} \boldsymbol{\Phi}_{k}^{i} \cdot\left[\epsilon_{i} \frac{\partial \mathbf{E}^{i}}{\partial t}-\nabla \times \mathbf{H}^{i}\right] d \mathbf{r}= \\
& \sum_{f=1}^{4} \int_{\partial \Omega_{i, f}} \boldsymbol{\Phi}_{k}^{i} \cdot\left[\hat{\mathbf{n}}_{i, f} \times\left(\mathbf{H}_{f}^{*}-\mathbf{H}^{i}\right)\right] d \mathbf{r} \\
& \int_{\Omega_{i}} \boldsymbol{\Psi}_{l}^{i} \cdot\left[\mu_{i} \frac{\partial \mathbf{H}^{i}}{\partial t}+\nabla \times \mathbf{E}^{i}\right] d \mathbf{r}= \\
& \sum_{f=1}^{4} \int_{\partial \Omega_{i, f}} \mathbf{\Psi}_{l}^{i} \cdot\left[\hat{\mathbf{n}}_{i, f} \times\left(\mathbf{E}^{i}-\mathbf{E}_{f}^{*}\right)\right] d \mathbf{r} .
\end{aligned}
$$


After a tedious mathematical operation, we can achieve the semidiscrete matrix equations as

$$
\begin{aligned}
\overline{\mathbf{M}}_{e}^{i} \frac{\partial \mathbf{e}^{i}}{\partial t}= & \overline{\mathbf{S}}_{e}^{i} \mathbf{h}^{i}+\sum_{f=1}^{4}\left(\overline{\mathbf{F}}_{\mathrm{ee}}^{\mathrm{ii}, f} \mathbf{e}_{f}^{i}+\overline{\mathbf{F}}_{\mathrm{ee}}^{\mathrm{ij}, f} \mathbf{e}_{f}^{j}\right. \\
& \left.+\overline{\mathbf{F}}_{\mathrm{eh}}^{\mathrm{ii}, f} \mathbf{h}_{f}^{i}+\overline{\mathbf{F}}_{\mathrm{eh}}^{\mathrm{ij}, f} \mathbf{h}_{f}^{j}\right)+\overline{\mathbf{F}}_{e}^{i, M_{s}} \\
\overline{\mathbf{M}}_{h}^{i} \frac{\partial \mathbf{h}^{i}}{\partial t}= & -\overline{\mathbf{S}}_{h}^{i} \mathbf{e}^{i}+\sum_{f=1}^{4}\left(\overline{\mathbf{F}}_{\mathrm{hh}}^{\mathrm{ii}, f} \mathbf{h}_{f}^{i}+\overline{\mathbf{F}}_{\mathrm{hh}}^{\mathrm{ij}, f} \mathbf{h}_{f}^{j}\right. \\
& \left.+\overline{\mathbf{F}}_{\mathrm{he}}^{\mathrm{ii}, f} \mathbf{e}_{f}^{i}+\overline{\mathbf{F}}_{\mathrm{he}}^{\mathrm{ij}, f} \mathbf{e}_{f}^{j}\right)+\overline{\mathbf{F}}_{h}^{i, M_{s}}
\end{aligned}
$$

where

$$
\begin{aligned}
& {\left[\overline{\mathbf{M}}_{e}^{i}\right]_{\mathrm{kl}}=\int_{\Omega_{i}} \boldsymbol{\Phi}_{k}^{i} \cdot \epsilon^{i} \boldsymbol{\Phi}_{l}^{i} d \mathbf{r}} \\
& {\left[\overline{\mathbf{M}}_{h}^{i}\right]_{\mathrm{kl}}=\int_{\Omega_{i}} \boldsymbol{\Psi}_{k}^{i} \cdot \mu^{i} \boldsymbol{\Psi}_{l}^{i} d \mathbf{r}} \\
& {\left[\overline{\mathbf{S}}_{e}^{i}\right]_{\mathrm{kl}}=\int_{\Omega_{i}} \boldsymbol{\Phi}_{k}^{i} \cdot \nabla \times \boldsymbol{\Psi}_{l}^{i} d \mathbf{r}} \\
& {\left[\overline{\mathbf{S}}_{h}^{i}\right]_{\mathrm{kl}}=\int_{\Omega_{i}} \boldsymbol{\Psi}_{k}^{i} \cdot \nabla \times \boldsymbol{\Phi}_{l}^{i} d \mathbf{r}} \\
& {\left[\mathbf{F}_{\mathrm{ee}}^{\mathrm{ii}, f}\right]_{\mathrm{kl}}=\frac{-1}{Z^{i}+Z_{f}^{j}} \int_{\partial \Omega_{i, f}} \boldsymbol{\Phi}_{k}^{i} \cdot \hat{\mathbf{n}}_{i, f} \times\left(\hat{\mathbf{n}}_{i, f} \times \Phi_{l}^{i}\right) d \mathbf{r}} \\
& {\left[\overline{\mathbf{F}}_{\mathrm{ee}}^{\mathrm{ij}, f}\right]_{\mathrm{kl}}=\frac{1}{Z^{i}+Z_{f}^{j}} \int_{\partial \Omega_{i, f}} \boldsymbol{\Phi}_{k}^{i} \cdot \hat{\mathbf{n}}_{i, f} \times\left(\hat{\mathbf{n}}_{i, f} \times \boldsymbol{\Phi}_{l}^{j, f}\right) d \mathbf{r}} \\
& {\left[\overline{\mathbf{F}}_{\mathrm{eh}}^{\mathrm{ii}, f}\right]_{\mathrm{kl}}=-\frac{Z_{f}^{j}}{Z^{i}+Z_{f}^{j}} \int_{\partial \Omega_{i, f}} \boldsymbol{\Phi}_{k}^{i} \cdot \hat{\mathbf{n}}_{i, f} \times \mathbf{\Psi}_{l}^{i} d \mathbf{r}} \\
& {\left[\overline{\mathbf{F}}_{\mathrm{eh}}^{\mathrm{ij}, f}\right]_{\mathrm{kl}}=\frac{Z_{f}^{j}}{Z^{i}+Z_{f}^{j}} \int_{\partial \Omega_{i, f}} \boldsymbol{\Phi}_{k}^{i} \cdot \hat{\mathbf{n}}_{i, f} \times \mathbf{\Psi}_{l}^{j, f} d \mathbf{r}} \\
& {\left[\overline{\mathbf{F}}_{e}^{i, M_{s}}\right]_{k}=\frac{-1}{Z^{i}+Z_{f}^{j}} \int_{\partial \Omega_{i, f g}} \boldsymbol{\Phi}^{i} \cdot\left(\hat{\mathbf{n}}_{i, f} \times \mathbf{M}_{s}\right) d \mathbf{r}} \\
& {\left[\overline{\mathbf{F}}_{\mathrm{hh}}^{\mathrm{ii}, f}\right]_{\mathrm{kl}}=\frac{-1}{Y^{i}+Y_{f}^{j}} \int_{\partial \Omega_{i, f}} \boldsymbol{\Psi}_{k}^{i} \cdot \hat{\mathbf{n}}_{i, f} \times\left(\hat{\mathbf{n}}_{i, f} \times \mathbf{\Psi}_{l}^{i}\right) d \mathbf{r}} \\
& {\left[\overline{\mathbf{F}}_{\mathrm{hh}}^{\mathrm{ij}, f}\right]_{\mathrm{kl}}=\frac{1}{Y^{i}+Y_{f}^{j}} \int_{\partial \Omega_{i, f}} \boldsymbol{\Psi}_{k}^{i} \cdot \hat{\mathbf{n}}_{i, f} \times\left(\hat{\mathbf{n}}_{i, f} \times \mathbf{\Psi}_{l}^{j, f}\right) d \mathbf{r}} \\
& {\left[\overline{\mathbf{F}}_{\mathrm{he}}^{\mathrm{ii}, f}\right]_{\mathrm{kl}}=\frac{Y_{f}^{j}}{Y^{i}+Y_{f}^{j}} \int_{\partial \Omega_{i, f}} \boldsymbol{\Psi}_{k}^{i} \cdot \hat{\mathbf{n}}_{i, f} \times \boldsymbol{\Phi}_{l}^{i} d \mathbf{r}} \\
& {\left[\overline{\mathbf{F}}_{\mathrm{he}}^{\mathrm{ij}, f}\right]_{\mathrm{kl}}=\frac{-Y_{f}^{j}}{Y^{i}+Y_{f}^{j}} \int_{\partial \Omega_{i, f}} \boldsymbol{\Psi}_{k}^{i} \cdot \hat{\mathbf{n}}_{i, f} \times \boldsymbol{\Phi}_{l}^{j, f} d \mathbf{r}} \\
& {\left[\overline{\mathbf{F}}_{h}^{i, M_{s}}\right]_{k}=\frac{-Y_{f}^{j}}{Y^{i}+Y_{f}^{j}} \int_{\partial \Omega_{i, f}} \mathbf{\Psi}_{k}^{i} \cdot \mathbf{M}_{s} d \mathbf{r} .}
\end{aligned}
$$

It should be noted that $\overline{\mathbf{F}}_{e}^{i, M_{s}}$ and $\overline{\mathbf{F}}_{h}^{i, M_{s}}$ are two column vectors and nonzero only over the ventilation slots and apertures of the shielding enclosure.
In this work, 12 edge vector basis functions (six constant tangential/linear normal (CT/LN) and six linear tangential/linear normal (LT/LN) basis functions, respectively) are used for both $\mathbf{E}$ and $\mathbf{H}$ in each mesh element, i.e., $n_{e}^{i}=12$ and $n_{h}^{i}=12$ for $i=1, \ldots, N$. Thus, the dimension of the matrix equations to be solved is 12 by 12 . In this study, the inversion of the mass matrices are precalculated and stored before starting the timemarching scheme.

To solve the semidiscrete matrix equations from (12) to (13), the fourth-order Runge-Kutta method is employed. For explicit time-marching scheme, the Courant-Friedrichs-Lewylike condition must be satisfied to ensure stability. In general, the time-step size $\delta t$ is determined in terms of the following condition [33], [36]:

$$
c_{0} \delta t \leq \min \left\{l_{\min } \sqrt{\epsilon_{r} \mu_{r}} / 4(p+1)^{2}\right\}
$$

where $c_{0}$ is the free-space light speed, $p$ is the order of basis function. In this study, first-order basis functions are used, thus $p=1$.

\section{TDBI Equation}

As a partial differential equation solver, DGTD method has to be truncated by artificial boundary conditions for open-space problems. At the truncation boundary $\partial \Omega$, the field values $\mathbf{E}^{j}$ and $\mathbf{H}^{j}$ in (8) and (9) used for incoming flux evaluation are not available. In this study, our previously developed hybrid DGTD and TDBI is employed by evaluating $\mathbf{E}^{j}$ and $\mathbf{H}^{j}$ using the equivalent electric current $\mathbf{J}_{i^{\prime}, f^{\prime}}^{\mathrm{h}}$ and magnetic current $\mathbf{J}_{i^{\prime}, f^{\prime}}^{\mathrm{e}}$ over a Huygens' surface enclosing the scatterer [33]. Namely,

$$
\begin{aligned}
\mathbf{E}_{f}^{j, \partial \Omega}(\mathbf{r}, t) & =\sum_{i^{\prime}} \sum_{f^{\prime}}\left[\mu_{0} \mathcal{L}_{i^{\prime}, f^{\prime}}\left(\mathbf{J}_{i^{\prime}, f^{\prime}}^{\mathrm{h}}\right)-\mathcal{K}_{i^{\prime}, f^{\prime}}\left(\mathbf{J}_{i^{\prime}, f^{\prime}}^{\mathrm{e}}\right)\right] \\
\mathbf{H}_{f}^{j, \partial \Omega}(\mathbf{r}, t) & =\sum_{i^{\prime}} \sum_{f^{\prime}}\left[\epsilon_{0} \mathcal{L}_{i^{\prime}, f^{\prime}}\left(\mathbf{J}_{i^{\prime}, f^{\prime}}^{\mathrm{e}}\right)+\mathcal{K}_{i^{\prime}, f^{\prime}}\left(\mathbf{J}_{i^{\prime}, f^{\prime}}^{\mathrm{h}}\right)\right]
\end{aligned}
$$

where the operators $\mathcal{L}_{i^{\prime}, f^{\prime}}$ and $\mathcal{K}_{i^{\prime}, f^{\prime}}$ are defined as

$$
\begin{aligned}
\mathcal{L}_{i^{\prime}, f^{\prime}}(\mathbf{J})= & -\frac{1}{4 \pi} \int_{\partial \Omega_{i^{\prime}, f^{\prime}}} \frac{\partial_{t} \mathbf{J}\left(\mathbf{r}^{\prime}, t-R / c\right)}{R} d \mathbf{r}^{\prime} \\
& +\frac{c^{2}}{4 \pi} \nabla \int_{\partial \Omega_{i^{\prime}, f^{\prime}}} \int_{0}^{t-R / c} \frac{\nabla^{\prime} \cdot \mathbf{J}\left(\mathbf{r}^{\prime}, t^{\prime}\right)}{R} d t^{\prime} d \mathbf{r}^{\prime} \\
\mathcal{K}_{i^{\prime}, f^{\prime}}(\mathbf{J})= & \frac{1}{4 \pi} \nabla \times \int_{\partial \Omega_{i^{\prime}, f^{\prime}}} \frac{\mathbf{J}\left(\mathbf{r}^{\prime}, t-R / c\right)}{R} d \mathbf{r}^{\prime}
\end{aligned}
$$

Since the equivalent Huygens' current sources are calculated by DGTD, all time-marching schemes are conducted explicitly, and no matrix inversion is involved. Thus, compared with traditional FEM-BI, the DGTD-BI scheme is more efficient.

\section{Comments and Discussions}

Due to the unavailability of the NF measurement equipment, only the simulated NF electric field data is used instead. In fact, we first use our DGTD solver to straightforwardly get the tangential components of the electric field over the slots and apertures. Then, the DGTD scheme introduced above is further applied to calculated the radiated emission outside the shielding enclosure. For practical situations, the time-step resolution $\delta t_{\text {meas }}$ used for NF sampling is usually different with 


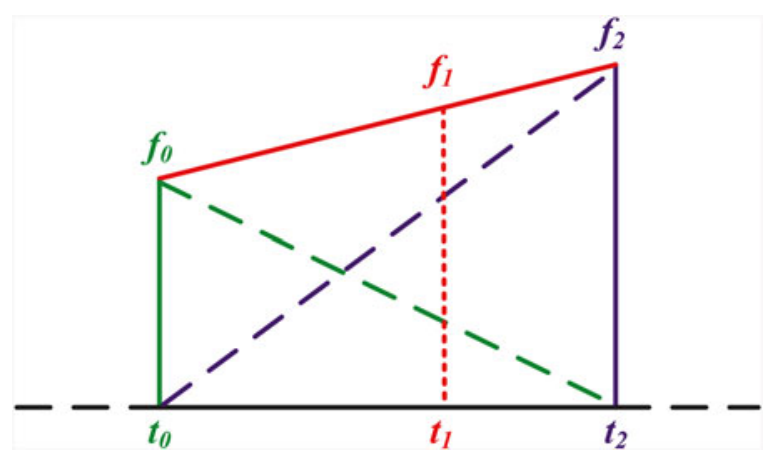

Fig. 2. Schematic illustration of the linear interpolation method. The function value $f_{1}$ is interpolated based on $f_{0}$ and $f_{2}$ and express as $f_{1}=P_{1} f_{0}+P_{0} f_{2}$, $P_{0}=\left(t_{1}-t_{0}\right) /\left(t_{2}-t_{0}\right)$ and $P_{1}=\left(t_{2}-t_{1}\right) /\left(t_{2}-t_{0}\right)$.

the time-step size $\delta t_{\mathrm{DG}}$ for the DGTD based solvers (see 14). Thus, the equivalent magnetic current $\mathbf{M}_{s}$ at $t=n \delta t_{\mathrm{DG}}$ on the right-hand side of (12) and (13) must be interpolated from the sampled NF electric field. To facilitate the interpolation scheme, the shifted polynomials $T(t)$ are deployed as the temporal basis functions [33]. That is,

$$
T(t)= \begin{cases}1-t /\left|\Delta t_{\text {meas }}\right| & -\Delta t_{\text {meas }} \leq t \leq \Delta t_{\text {meas }} \\ 0 & \text { otherwise. }\end{cases}
$$

An schematic illustration is shown in Fig. 2.

To include the influence of the practical measurement errors on the accuracy of the proposed method, the artificial noise is added to the simulated NF data. The amount of the noise is gauged by the signal-to-noise ratio (SNR). The results obtained from both the noise-free and noise-contaminated NF data are presented and compared to verify the robustness of the developed approach.

\section{NUMERICAL RESULTS}

In this part, representative examples are provided to validate the proposed method, including a Hertzian dipole in the free space and two PCBs in shielding enclosures.

\section{A. Hertzian Dipole in Free Space}

With the purpose to demonstrate the accuracy of our approach, we consider a z-directed electric Hertzian dipole in free space as the first example. The tangential components of the electric field over a spherical surface $\left(r_{\text {meas }}=0.1 \mathrm{~m}\right)$ enclosing the dipole are computed analytically by

$$
\begin{aligned}
E_{\theta}= & \frac{\eta_{0} \sin (\theta)}{4 \pi R}\left[\frac{1}{c_{0}} \partial_{t} \mathrm{~g}\left(t-R / c_{0}\right)+\frac{\mathrm{g}\left(t-R / c_{0}\right)}{R}+\right. \\
& \left.\times \frac{c_{0}}{R^{2}} \int_{0}^{t-R / c_{0}} \mathrm{~g}(\tau) d \tau\right]
\end{aligned}
$$

where $\mathrm{g}(t)=\frac{2}{15}\left(t-t_{0}\right) \exp \left(-\left(t-t_{0}\right)^{2} / t_{m}^{2}, t_{m}=1.27 \mathrm{~ns}\right.$ and $t_{0}=5 t_{m}$.

For this example, the time-step sizes for the NF sampling and DGTD are $\delta t_{\text {meas }}=10 \mathrm{ps}$ and $\delta t_{\mathrm{DG}}=1.2 \mathrm{ps}$, respectively. And, the time-marching step-size for TDBI is $\delta t_{\mathrm{BI}}=21.1 \mathrm{ps}$. The computed electric fields by the proposed approach at

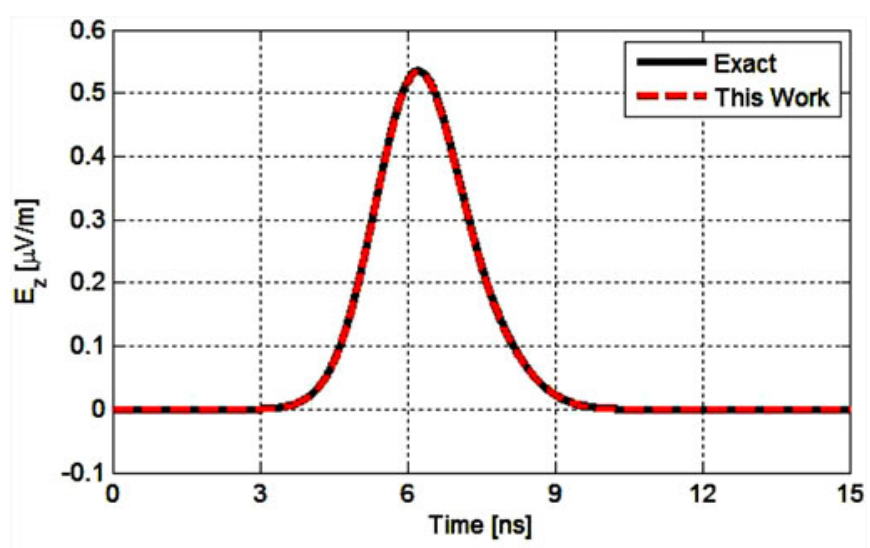

(a)

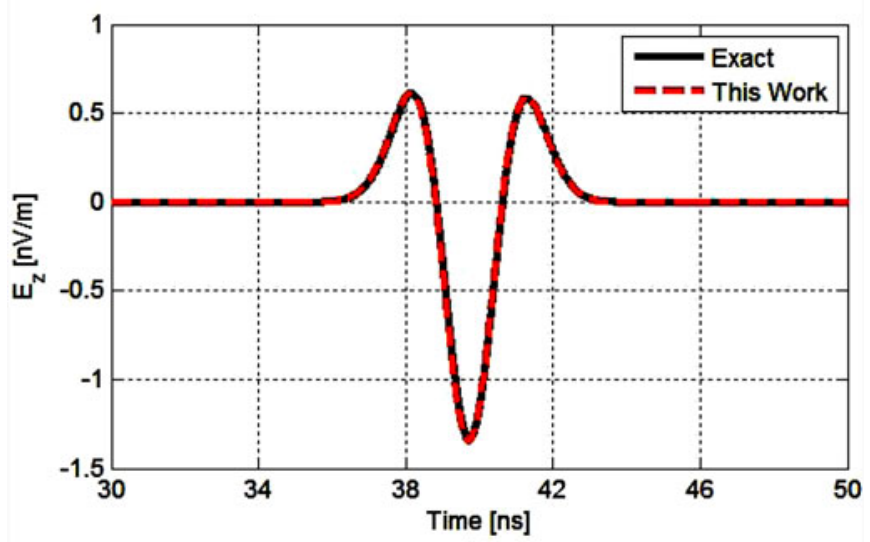

(b)

Fig. 3. Calculated electric field component $E_{z}$ at $\mathbf{r}_{0}=0.12 \hat{\mathbf{y}} \mathrm{m}$ (a) and $\mathbf{r}_{0}=10 \hat{\mathbf{y}} \mathrm{m}$ (b). For comparison, the analytical results by (18).

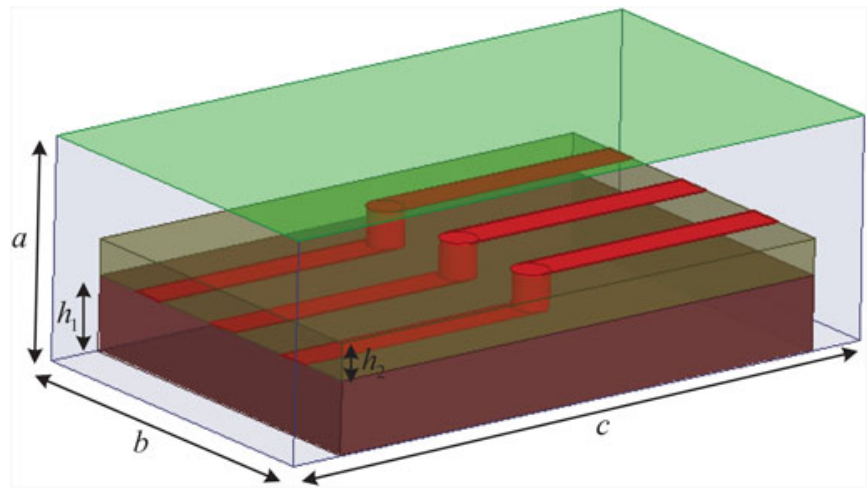

Fig. 4. Two-layer PCB with microstrip traces and vertical vias placed into a shielding enclosure with top-opened (denoted by the green surface, where the $\mathrm{NF}$ sampling is conducted). The parameters of the shielding box are defined as: $a=6 \mathrm{~cm}, b=10 \mathrm{~cm}, c=18 \mathrm{~cm}, h_{1}=2 \mathrm{~cm}$, and $h_{2}=1 \mathrm{~cm}$.

$\mathbf{r}=(0,0.12,0) \mathrm{m}$ and $(0,10,0) \mathrm{m}$ are plotted in Fig. 3. For comparison, the analytical solutions are also provided. It is noted that excellent agreements are observed both in the NF and FF region.

\section{B. PCB Board in a Top-Opened Shielding Enclosure}

To further validate our approach, in the second example, the radiation from a PCB placed in a shielding box without top covers is investigated, as shown in Fig. 4. The PCB has two 


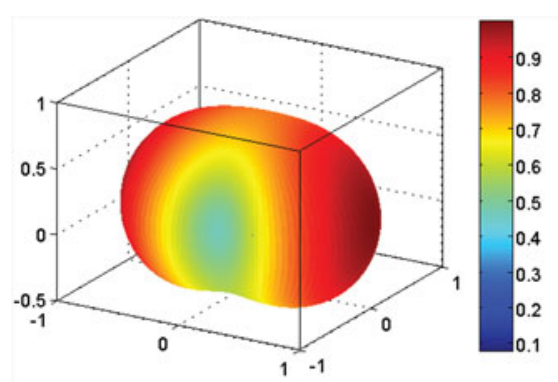

(a)

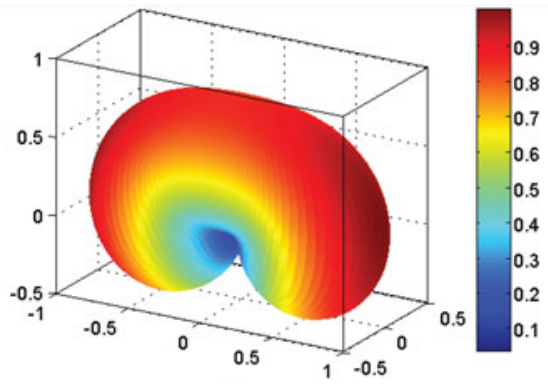

(b)

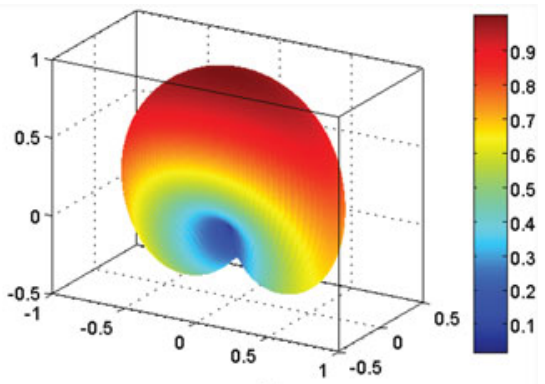

(c)

Fig. 5. Calculated FF radiation patterns by the proposed method at $f_{1}=0.5 \mathrm{GHz}(\mathrm{a}), f_{2}=0.75 \mathrm{GHz}$ (b), and $f_{3}=1.0 \mathrm{GHz}$ (c).

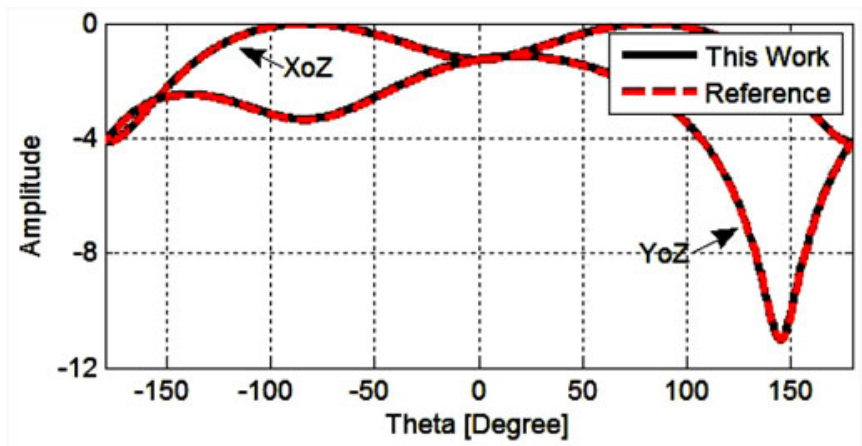

(a)

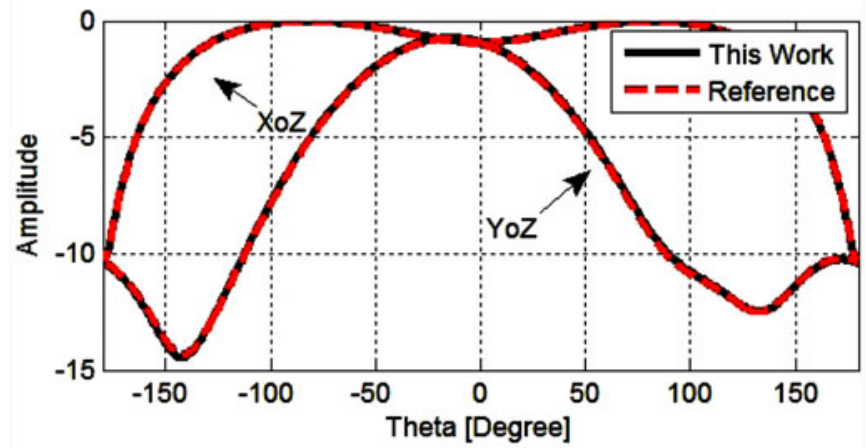

(b)

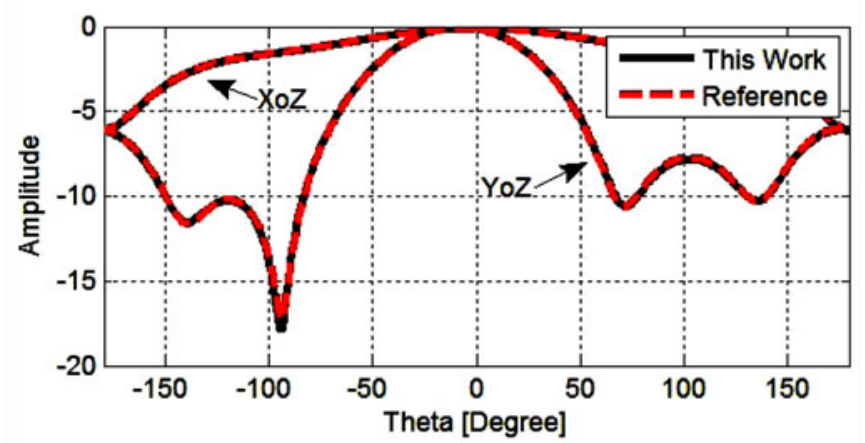

(c)

Fig. 6. FF patterns in $x o y$ and yoz planes corresponding to the 3-D patterns in Fig. 5.

dielectric layers with thickness denoted as $h_{1}$ and $h_{2}$. The relative electric permittivities are 3.0 and 2.0, respectively. Three signal lines with six ports defined at the left and right side of the package are distributed over the two-layered PCB. In our simulation, the left three ports are stimulated by three lumped voltage sources with $R_{s}=50 \Omega$.

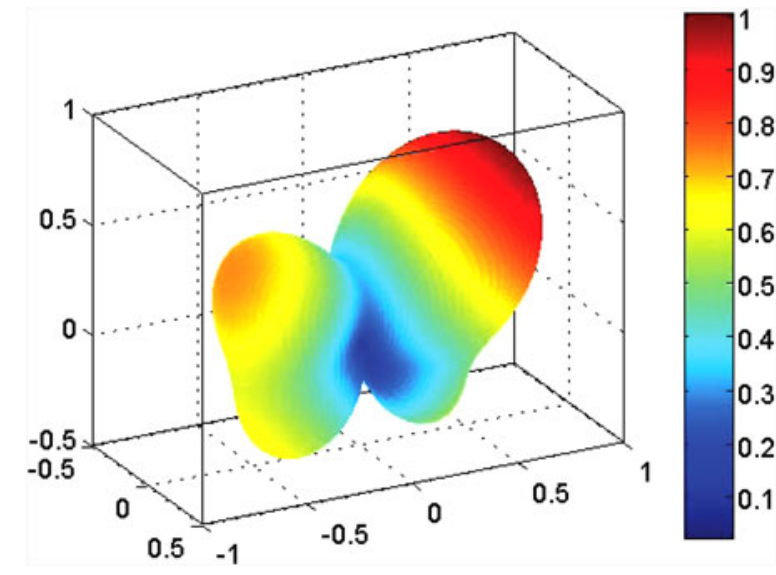

Fig. 7. Calculated FF pattern at $f=1.0 \mathrm{GHz}$ by the proposed approach.

First, we assume that the right three ports are shorted to the ground (a PEC boundary condition), and the NF electric fields over the top cover are obtained by time-domain simulation, and the corresponding sampling time-step size is around $4.62 \mathrm{~ns}$. Next, the above proposed scheme is facilitated to calculate the radiated emission outside the shielding box. For this step, the time-step sizes for DGTD and TDBI are $\delta t_{\mathrm{DG}}=0.59 \mathrm{~ns}$ and $\delta t_{\mathrm{BI}}=13.61 \mathrm{~ns}$, respectively. In Fig. 5, the FF radiation patterns at frequency $f=0.5,0.75$, and $1.0 \mathrm{GHz}$ are presented. For comparison, the simulated counterparts by direct full-wave simulation are also shown in Fig. 6 . Obviously, very good agreements are achieved. It is noted that the forward radiation becomes stronger and the beam is more narrow with the frequency increasing.

Second, the situation when the right three ports are floating in the space are considered. Similarly, the simulated NF fields over the top cover are used as the equivalent magnetic current $\mathbf{M}_{s}\left(\mathbf{r}^{\prime}, t\right)$. For this example, only the 3-D FF radiation pattern at $f=1.0 \mathrm{GHz}$ are calculated and shown in Fig. 7, while the 2-D FF patterns in $x o z$ and yoz planes at $f=0.5,0.75$, and $1.0 \mathrm{GHz}$ are plotted in Fig. 8. The simulated references are also given for accuracy comparison. Clearly, good agreements are noted.

\section{PCB in a Shielding Enclosure With Slots}

In the last example, a PCB placed in a shielding enclosure with two ventilation slots is demonstrated, as shown in Fig. 9. The thickness and the relative electric permittivity of the substrate 


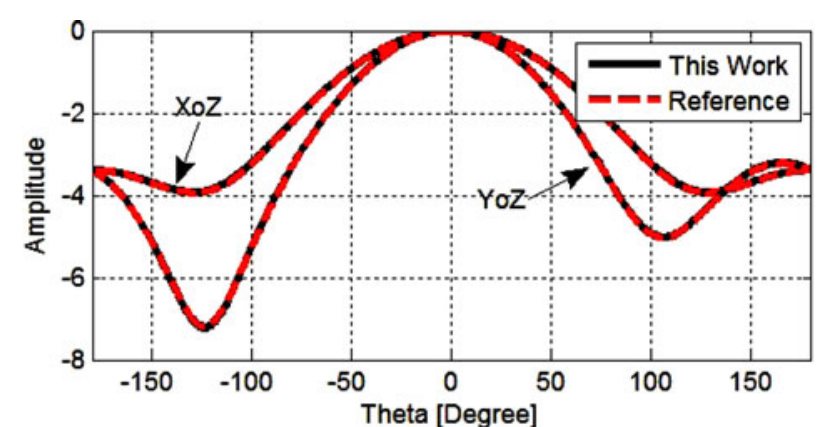

(a)

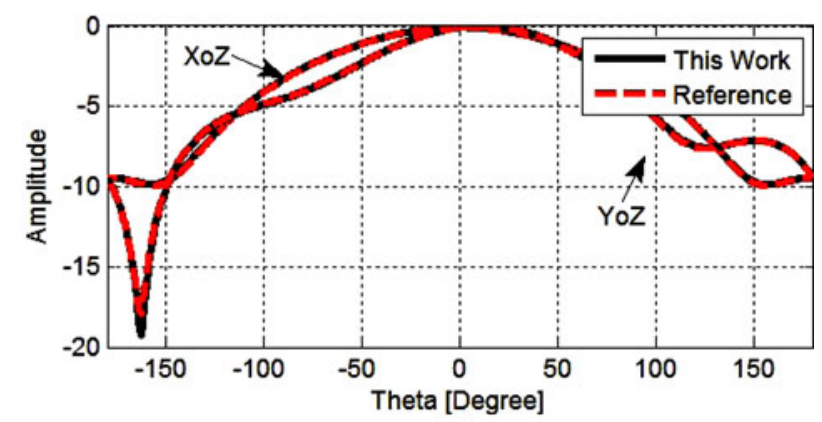

(b)

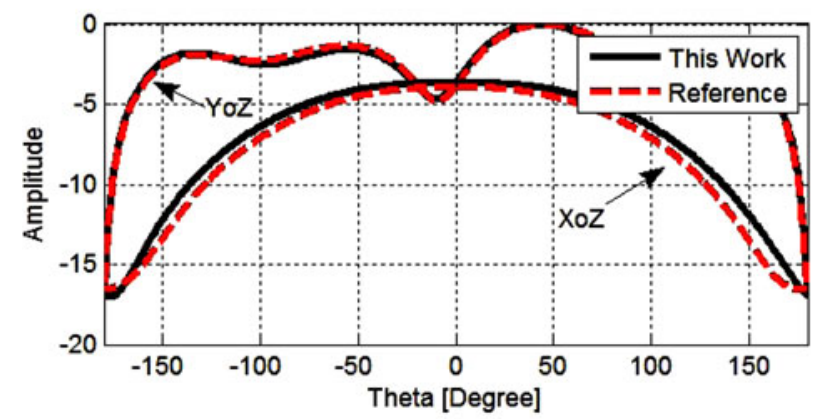

(c)

Fig. 8. FF patterns in $x o y$ and $y o z$ planes corresponding to the 3-D patterns in Fig. 7.

are 0.02 and $2.2 \mathrm{~m}$, respectively. The left port is driven by a lumped voltage source [38] defined as

$$
\begin{aligned}
V_{s}(t)= & e^{-\alpha_{1}^{2}\left(f_{2}-f_{1}\right)^{2}\left(t-\frac{\alpha_{2}}{f_{2}-f_{1}}\right)^{2}} \\
& \times \cos \left[2 \pi \frac{f_{1}+f_{2}}{2}\left(t-\frac{\alpha_{2}}{f_{2}-f_{1}}\right)\right]
\end{aligned}
$$

with $f_{1}=0.4 \mathrm{GHz}, f_{2}=1.6 \mathrm{GHz}, \alpha_{1}=1.035$ and $\alpha_{2}=$ 2.539 , and the right port is loaded with a resistor $R_{0}=50 \Omega$. To facilitate the proposed algorithm, the temporal tangential electric fields over the slots are obtained via simulation with sampling time resolution $\delta t_{\text {meas }}=5.64$ ps. Due to the strong cavity resonance, the sampling is conducted over a long time duration around $176 \mathrm{~ns}$. Then, the proposed strategy is applied to characterize the radiation outside the shielding box. The time-step sizes for DGTD and TDBI are $\delta t_{\mathrm{DG}}=0.58 \mathrm{ps}$ and $\delta t_{\mathrm{BI}}=19.72 \mathrm{ps}$, respectively. In Fig. 10, the recorded electric field at $(0,0,0.12)$ $\mathrm{m}$ is plotted. The reference solution is also provided. It can be seen that the fields take much time to degrade because of the cavity resonance. In Fig. 11, the computed 3-D FF patterns at

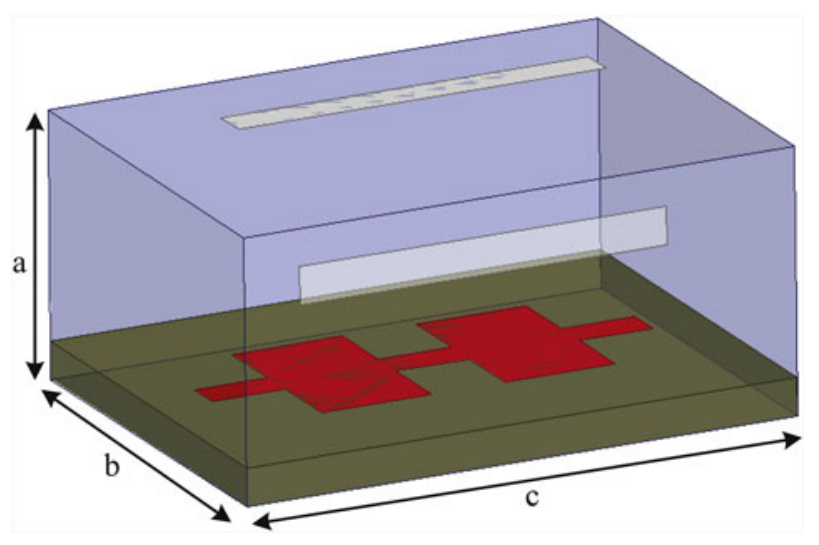

Fig. 9. PCB in a shielding enclosure with parameters defined as $a=0.14 \mathrm{~m}$, $b=0.22 \mathrm{~m}$, and $c=0.3 \mathrm{~m}$. The width and length of the two slots are 0.02 and $0.2 \mathrm{~m}$, respectively.

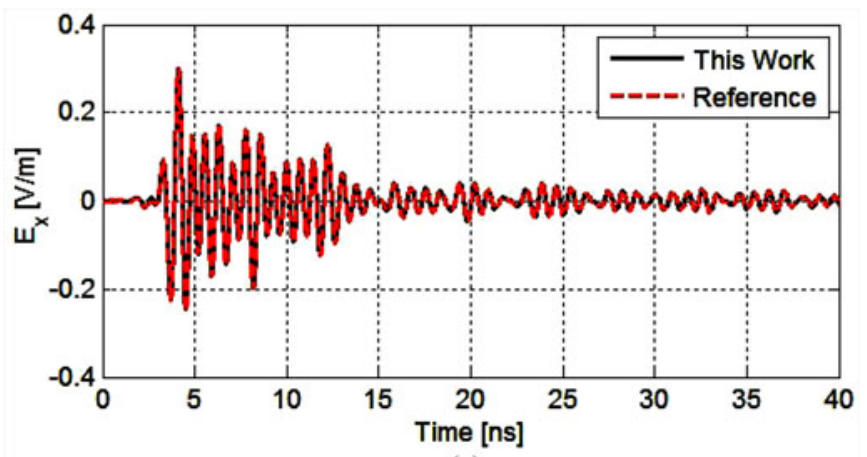

(a)

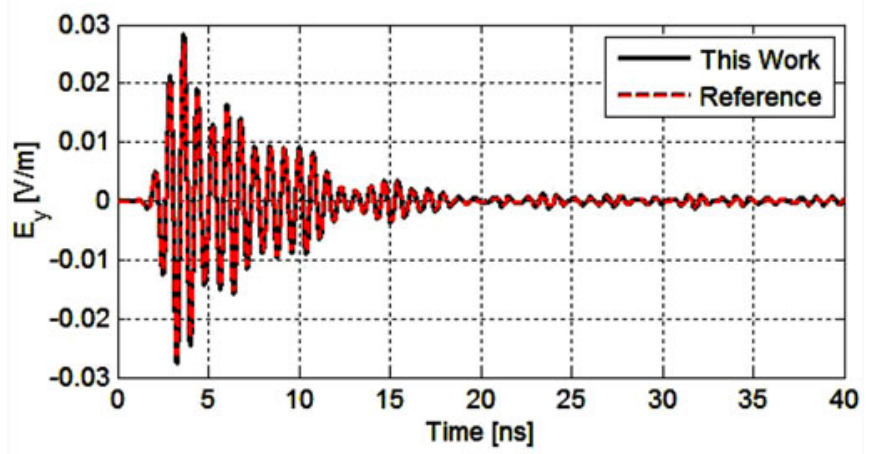

(b)

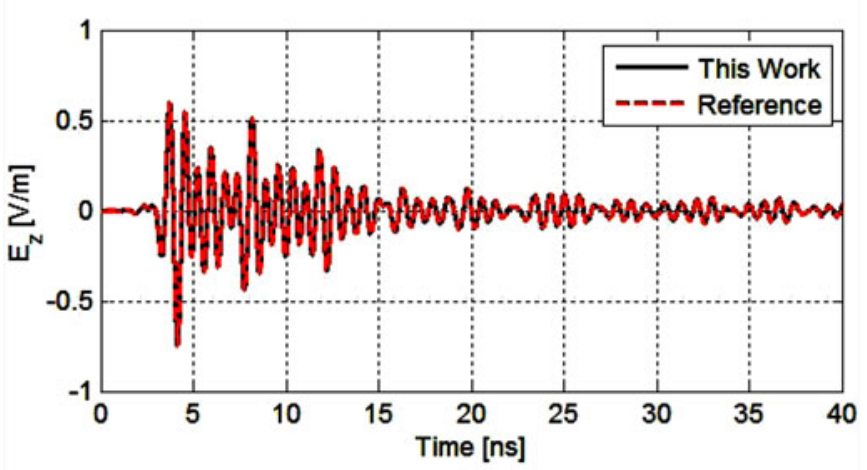

(c)

Fig. 10. $x, y$, and $z$-components of the electric field at $\mathbf{r}^{\prime}=0.12 \hat{\mathbf{z}}$ as the function of time $t$. 

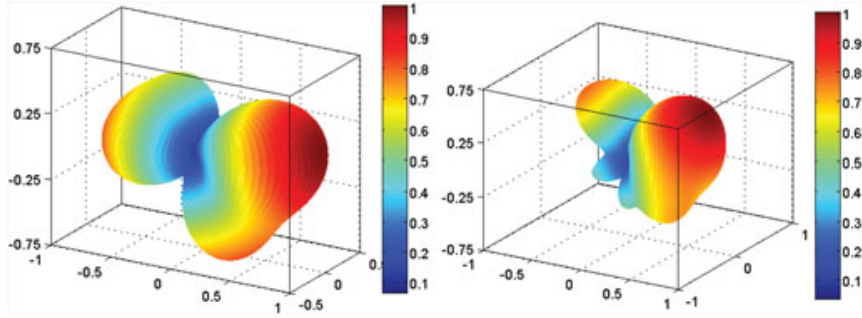

Fig. 11. Obtained FF 3-D patterns at frequencies $f_{1}=0.5 \mathrm{GHz}$ (left) and $f_{2}=1.25 \mathrm{GHz}$ (right).

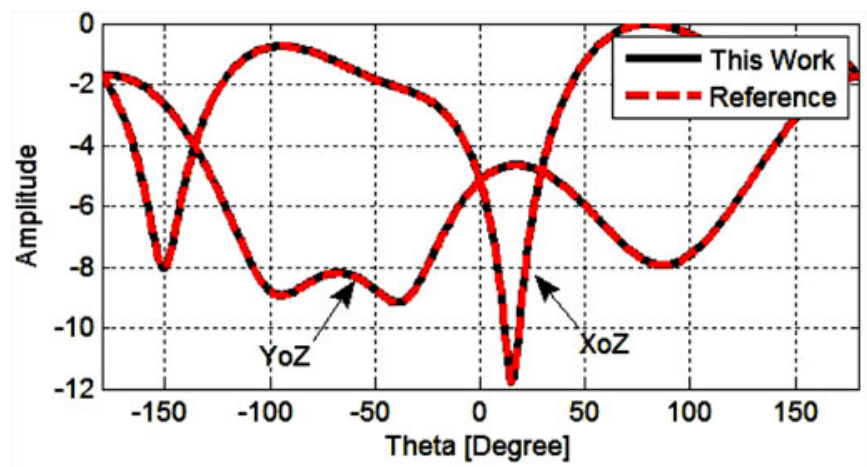

(a)

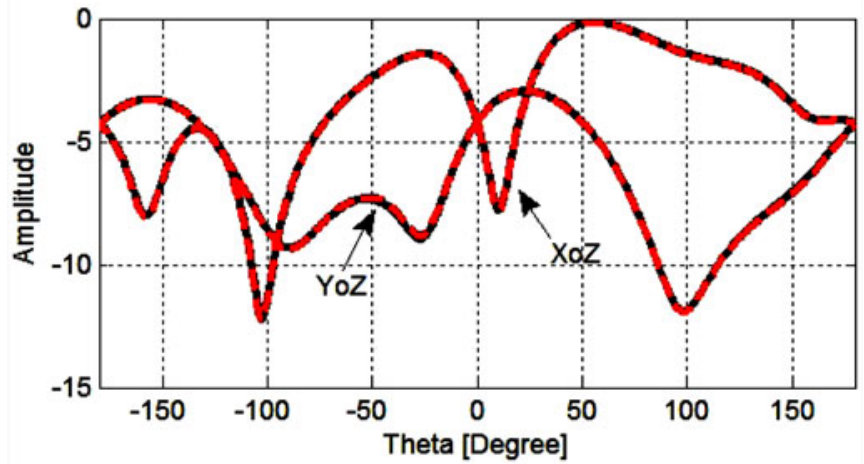

(b)

Fig. 12. 2-D FF patterns in $x o z$ and yoz planes corresponding to the 3-D patterns in Fig. 11.

$f=0.5$ and $1.25 \mathrm{GHz}$ are shown. To compare with the reference results, the 2-D patterns in $x o z$ and yoz planes are plotted in Fig. 12. Again, very good agreements between the proposed results and the reference solution are reached.

To characterize the cavity resonance enhanced radiation, the radiated energy normalized to the source power is calculated by

$$
\mathrm{P}_{r}(\omega)=\mathrm{R}_{s} \times \frac{\oint_{S} \mathbf{E}(\omega) \times \mathbf{H}^{*}(\omega) d S}{\left\|\mathbf{V}_{\mathrm{s}}(\omega) \times \mathbf{V}_{\mathrm{S}}^{*}(\omega)\right\|}
$$

where $\mathrm{R}_{s}=50 \Omega$ denotes the source impedance, and the integration is conducted over a surface $S$ enclosing the shielding box. The calculated result from 0.5 to $1.2 \mathrm{GHz}$ is presented in Fig. 13, which agrees to the solution obtained by direct simulation very well. Based on the result, it is interesting to found that the cavity resonance has significant influence on the radiation intensity.

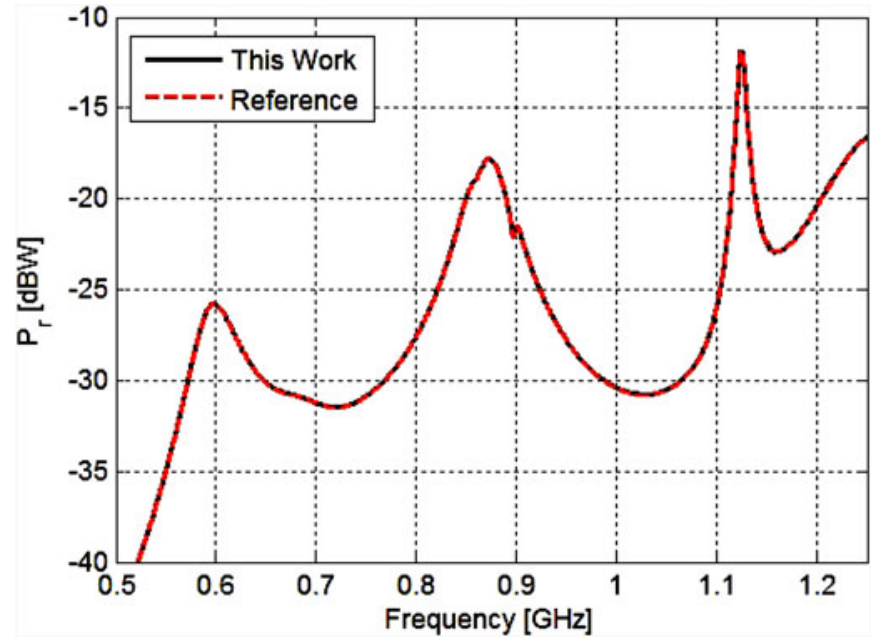

Fig. 13. Radiated power versus frequency by the proposed algorithm and the reference result obtained by straightforward simulation.

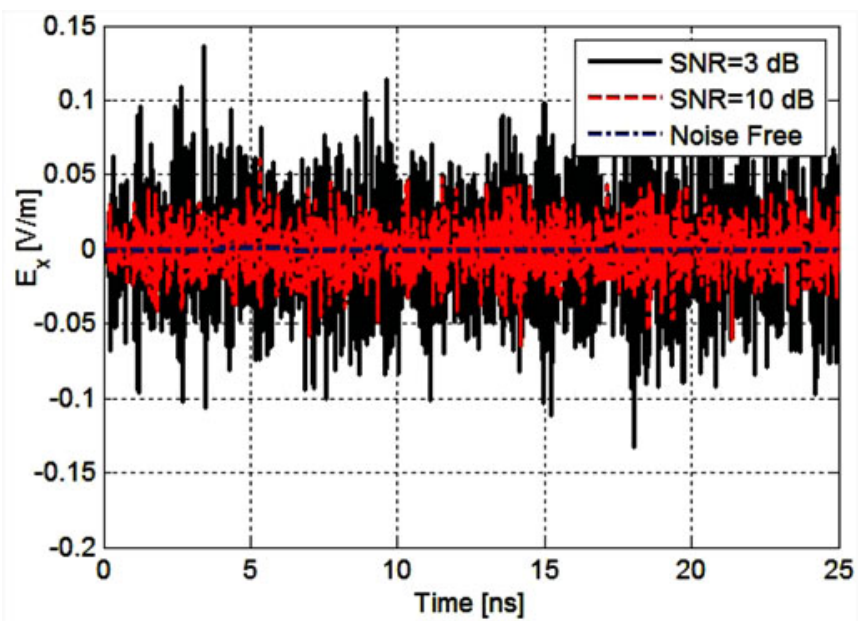

(a)

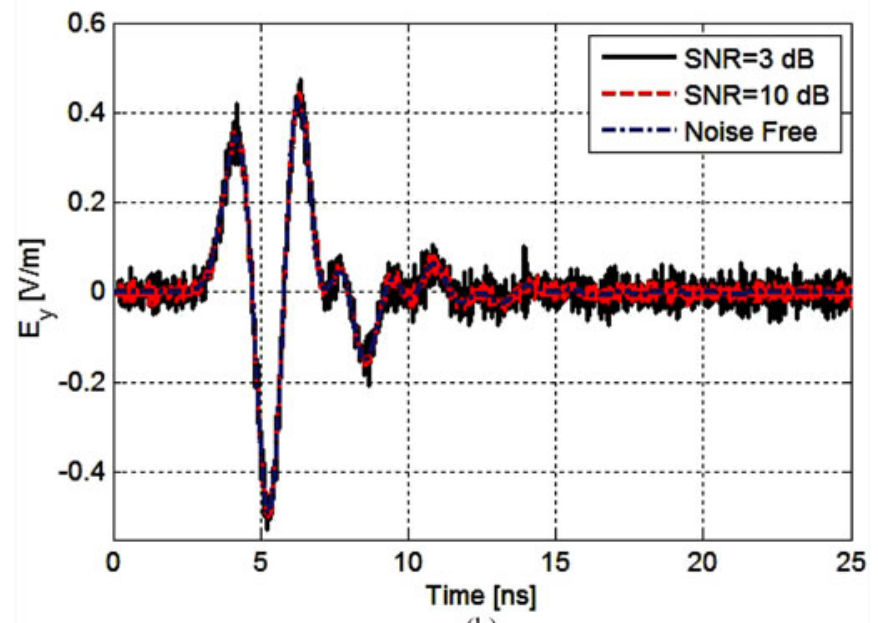

(b)

Fig. 14. Calculated NF electric field using noise-contaminated data with different SNRs by the proposed method. The $x$ (a) and $y$ (b) components of near electric-field. 


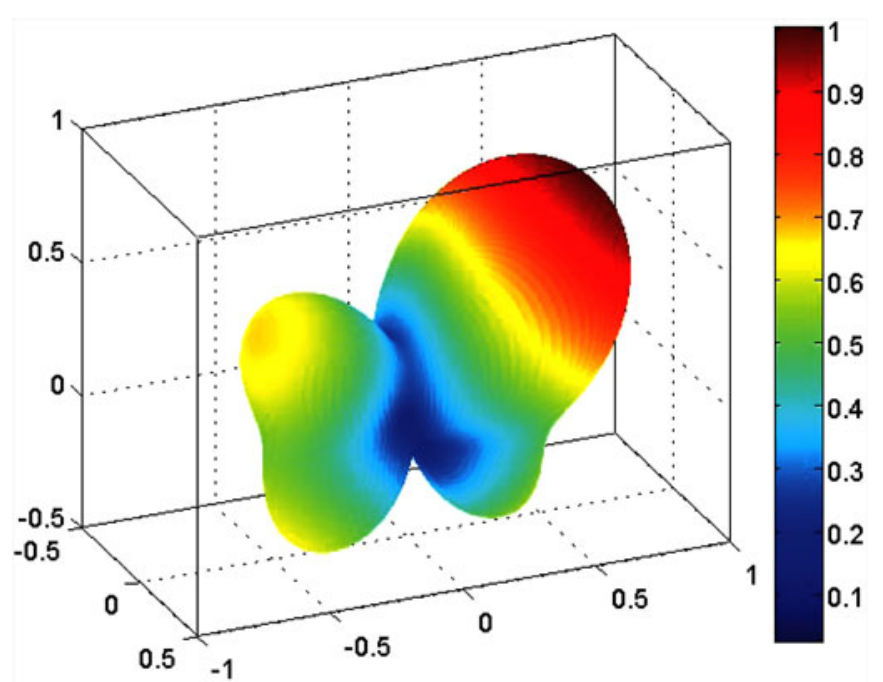

(a)

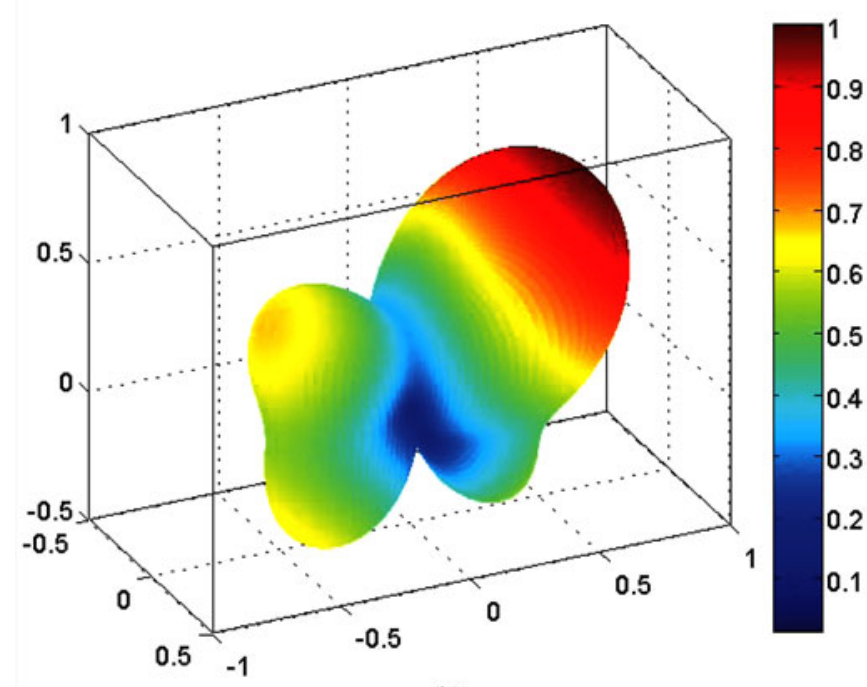

(b)

Fig. 15. FF pattern calculated by noise data with $\mathrm{SNR}=3 \mathrm{~dB}$ (a) and $\mathrm{SNR}=10 \mathrm{~dB}(\mathrm{~b})$.

\section{Investigation of Noise Influence}

For practical NF scanning situation, the reliability of the measurement data is highly affected by uncertainty faults including probe-positioning error, the dynamics of the probe, background noise, and the influence of cables, etc. Thus, it is necessary to investigate the robustness of the proposed approach if the noisepolluted NF data was employed. To achieve this objective, we artificially add noise with different SNR to the simulated NF data. The benchmark in Section III-B is revisited again, but only the open-circuit case is considered. First, the NF data at $\mathbf{r}^{\prime}=0.1 \hat{\mathbf{z}}$ is calculated corresponding to different SNRs, as shown in Fig. 14. Significant influence on the NF data is observed. To study the influence on the FF patterns, in Fig. 15, the FF radiation pattern at $1.0 \mathrm{GHz}$ using noise data is presented. Compared with the noise-free obtained FF pattern in Fig. 7, it is interestingly found that the noise has trivial effects on the FF patterns compared with the NF data.

\section{CONCLUSION}

In this paper, a time-domain NF-FF transformation approach is proposed to model the radiated emissions from the electronic systems placed in shielding box using only near electric field over the ventilation slots and apertures. Because of unavailability of the analytical DGF in the presence of an arbitrary-shaped PEC box, the DGTD-based full-wave numerical algorithm is employed by considering the original radiation problem as a scattering issue from the PEC-filled shielding enclosure with incident wave generated by the equivalent magnetic currents $\mathbf{M}_{s}=-\hat{\mathbf{n}} \times \mathbf{E}$ over the slots and apertures of the enclosure. Both analytical and numerical benchmarks are given to verify the developed method. The robustness of the approach is further verified by using noise-polluted NF data.

\section{REFERENCES}

[1] T. Sudo, H. Sasaki, N. Masuda, and J. L. Drewniak, "Electromagnetic interference (EMI) of system-on-package (SOP)," IEEE Trans. Adv. Packag., vol. 27, no. 2, pp. 304-314, May 2004.

[2] P. Li and L. J. Jiang, "A rigorous approach for the radiated emission characterization based on the spherical magnetic field scanning," IEEE Trans. Electromagn. Compat., vol. 56, no. 3, pp. 683-690, Jun. 2014.

[3] P. Li and L. J. Jiang, "A novel characterization method of the radiation emission for electromagnetic compatibility," in Proc. IEEE Int Symp. Electromagn. Compat., Long Beach, CA, USA, Aug. 2011, pp. 264-269.

[4] H. W. Shim and T. D. Hubing, "A closed-form expression for estimating radiated emissions from the power planes in a populated printed circuit board," IEEE Trans. Electromagn. Compat., vol. 48, no. 1, pp. 74-81, Feb. 2006.

[5] M. P. May, A. Taflove, and J. Baron, "FD-TD modeling of digital signal propagation in 3-D circuits with passive and active loads," IEEE Trans. Microw. Theory Techn., vol. 42, no. 8, pp. 1514-1523, Aug. 1994.

[6] M. Ha, K. Srinivasan, and M. Swaminathan, "Transient chip-package cosimulation of multiscale structures using the Laguerre-FDTD scheme," IEEE Trans. Compon. Packag. Technol., vol. 32, no. 4, pp. 816-830, Nov. 2009.

[7] K. Srinivasan, P. Yadav, A. E. Engin, M. Swaminathan, and M. Ha, "Fast EM/circuit transient simulation using Laguerre equivalent circuit (SLeEC)," IEEE Trans. Electromagn. Compat., vol. 51, no. 3, pp. 756762, Aug. 2009.

[8] Y. Shao, Z. Peng, and J. F. Lee, "Full-wave real-life 3-D package signal integrity analysis using nonconformal domain decomposition method," IEEE Trans. Microw. Theory Techn., vol. 59, no. 2, pp. 230-241, Feb. 2011.

[9] Z. G. Qian and W. C. Chew, "Fast full-wave surface integral equation solver for multiscale structure modeling," IEEE Trans. Antennas Propag., vol. 57, no. 11, pp. 3594-3601, Nov. 2009.

[10] V. P. Kodali, Engineering Electromagnetic Compatability, 2nd ed. Piscataway, NJ, USA: IEEE Press, 2001.

[11] P. K. Saha and J. Dowling, "Reliable prediction of EM radiation from a PCB at the design stage of electronic equipment," IEEE Trans. Electromagn. Compat., vol. 40, no. 2, pp. 166-174, May 1998.

[12] A. Ludwig, "Near-field far-field transformations using spherical wave expansions," IEEE Trans. Antennas Propag., vol. 19, no. 2, pp. 214-220, Mar. 1971

[13] W. M. Leach and D. T. Paris, "Probe compensated near-field measurements on a cylinder," IEEE Trans. Antennas Propag., vol. 21, no. 4, pp. 435-445, Jul. 1973.

[14] J. J. H. Wang, "An examination of the theory and practices of planar near-field measurement," IEEE Trans. Antennas Propag., vol. 36, no. 6 , pp. 746-753, Jun. 1988.

[15] P. Li and L. J. Jiang, "The far field transformation for the antenna modeling based on spherical electric field measurements," Progr. Electromagn. Res. vol. 123, pp. 243-261, 2012. 
[16] P. Peter and T. K. Sakar, "Planar near-field to far-field transformation using an equivalent magnetic current approach," IEEE Trans. Antennas Propag., vol. 40, no. 11, pp. 1348-1356, Nov. 1992.

[17] T. K. Sakar and A. Taaghol, "Near-field to near/far-field transformation for arbitrary near-field geometry utilizing an equivalent electric current and MoM," IEEE Trans. Antennas Propag., vol. 47, no. 3, pp. 566-573, Mar. 1999.

[18] J. L. A. Quijanoand and G. Vecchi, "Improved-accuracy source reconstruction on arbitrary 3-D surfaces," IEEE Antennas Wireless Propag. Lett., vol. 8, pp. 1046-1049, Sep. 2009.

[19] Y. Alvarez, F. Las-Heras, and M. R. Pino, "Reconstruction of equivalent currents distribution over arbitrary three-dimensional surfaces based on integral equation algorithms," IEEE Trans. Antennas Propag., vol. 55, no. 12, pp. 3460-3468, Nov. 2007.

[20] Y. Alvarez, M. Rodriguez, Fernando Las-Heras, and M. M. Hernando, "On the use of the source reconstruction method for estimation radiated EMI in electronics circuits," IEEE Trans. Inst. Meas., vol. 59, no. 12, pp. 3174-3183, Dec. 2010.

[21] P. Li and L. J. Jiang, "Source reconstruction method-based radiated emission characterization for PCBs," IEEE Trans. Electromagn. Compat., vol. 55, no. 5, pp. 933-940, Oct. 2013.

[22] P. Li, Y. Li, L. J. Jiang, and J. Hu, "A wide-band equivalent source reconstruction method exploiting the Stoer-Bulirsch algorithm with the adaptive frequency sampling," IEEE Trans. Antennas Propag., vol. 61, no. 10, pp. 5338-5343, Oct. 2013.

[23] S. M. Rao, D. R. Wilton, and A. W. Glisson, "Electromagnetic scattering by surfaces of arbitrary shape," IEEE Trans. Antennas Propag., vol. 30, no. 3, pp. 409-418, May 1982.

[24] J. R. Regue, M. Ribo, J. M. Garrell, and A. Martin, "A genetic algorithm based method for source identification and far-field radiated emissions prediction from near-field measurements for PCB characterization," IEEE Trans. Electromagn. Compat., vol. 43, no. 4, pp. 520-530, Nov. 2001.

[25] X. Tong, D. W. P. Thomas, A. Nothofer, P. Sewell, and C. Christopoulos, "Modeling electromagnetic emissions from printed circuit boards in closed environments using equivalent dipoles," IEEE Trans. Electromagn. Compat., vol. 52, no. 2, pp. 462-470, May 2010.

[26] S. R. Rengarajan and Y. Rahmat-Samii, "The field equivalence principle: Illustration of the establishment of the non-intuitive null fields," IEEE Trans. Antennas Propog., vol. 42, no. 4, pp. 122-128, Aug. 2000.

[27] J. S. Hesthaven and T. Warburton, Nodal Discontinuous Galerkin Methods. Berlin, Germany: Springer, 2008.

[28] P. Li and L. J. Jiang, "A hybrid electromagnetics-circuit simulation method exploiting discontinuous Galerkin finite element time domain method," IEEE Microw. Wireless Compon. Lett., vol. 23, no. 3, pp. 113-115, Mar. 2013.

[29] P. Li and L. J. Jiang, "Integration of arbitrary lumped multiport circuit networks into discontinuous Galerkin time-domain analysis," IEEE Trans. Microw. Theory Techn., vol. 61, no. 7, pp. 2525-2534, Jul. 2013.

[30] X. Gao, J. Fan, Y. Zhang, H. Kajbaf, and D. Pommerenke, "Farfield prediction using only magnetic near-field scanning for EMI test," IEEE Trans. Electromagn. Compat., vol. 56, no. 6, pp. 1335-1343, Dec. 2014.

[31] K. Sankaran, "Accurate domain truncation techniques for time-domain conformal methods," Ph.D. dissertation, Dept. Inf. Technol. Elect. Eng., ETH Zurich, Zurich, Switzerland, 2007.

[32] J. M. Jin, The Finite Element Method in Electromagnetics, 2nd ed. New York, NY, USA: Wiley, 2003.

[33] P. Li, Y. Shi, L. J. Jiang, and H. Bağcı, "A hybrid time-domain discontinuous galerkin-boundary integral method for electromagnetic scattering analysis," IEEE Trans. Antennas Propag., vol. 62, no. 5, pp. 2841-2846, May 2014.

[34] J. A. Kong, Electromagnetic Wave Theory. Cambridge, MA, USA: EMW Publishing, 2008.

[35] P. Li, "Numerical methodologies for electromagnetic parasitic system modeling and simulation," Ph.D. dissertation, Dept. Elect. Electron. Eng., The Univ. of Hong Kong, Hong Kong, 2014.
[36] S. D. Gedney, C. Luo, J. A. Roden, R. D. Crawford, B. Guernsey, J. A. Miller, T. Kramer, and E. W. Lucas, "The discontinuous Galerkin finite element time-domain method solution of Maxwell's equations," $J$. Appl. Comput. Electromagn. Society, vol. 24, no. 2, pp. 129-142, Apr. 2009.

[37] J. Xiong, "Computation electromagnetics for microstrip and MEMS structures," Ph.D. dissertation, Univ. Illinois Urbana-Champaign, Champaign, IL, USA, 2010.

[38] M. Li, K. P. Ma, D. M. Hockanson, J. L. Drewniak, T. H. Hubing, and T. P. V. Doren, "Numerical and experimental corroboration of an FDTD thinslot model for slots near corners of shielding enclosures," IEEE Trans. Electromagn. Compat., vol. 39, no. 3, pp. 225-232, Aug. 1997.

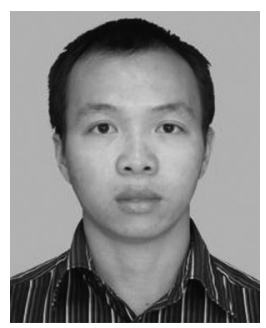

Ping Li (S'12-M'15) has been working toward the $\mathrm{Ph} . \mathrm{D}$. degree with the Center of Electromagnetics and Optics, University of Hong Kong since 2010.

$\mathrm{He}$ has authored more than ten journal papers on IEEE TRANSACTIONS ON MICROWAVE THEORY AND TECHNIQUES, IEEE TRANSACTIONS ON ANTENNAS and PROPAGATION, IEEE TRANSACTIONS ON ELECTROMAGNETIC AND COMPATIBILITY, IEEE TRANSACTION ON COMPONENTS, PACKAGING, AND MANUFACTURING TECHNOLOGIES, etc. His research interests include the near-field to far-field transformation techniques, phaseless equivalent source reconstruction methods, discontinuous Galerkin time-domain method, and uncertainty quantification for large scale electromagnetic systems.

Dr. Li's paper was selected as the Finalist paper in 29th International Review of Progress in Applied Computational Electromagnetics and 2014 International Symposium on Electromagnetic Compatibility, and he won the Best Student Paper Award in 12th International Workshop on Finite Elements for Microwave Engineering. He was the Reviewer of the IEEE TRANSACTION MicrowaVE THEORY AND TECHNIQUES, the IEEE TRANSACTION ON ANTENNAS AND PROPagation, the IEEE ANTENNAS AND Wireless Propagation LetTers, the PROCEEDINGS OF IEEE, Progress in Electromagnetics Research Letters, the Journal of Electromagnetic Waves and Applications, the Journal of ACES. He received the Postgraduate Engineering Fellowship from 2010. He is listed in Marquis Who's Who in the World, 32nd Edition, 2015.

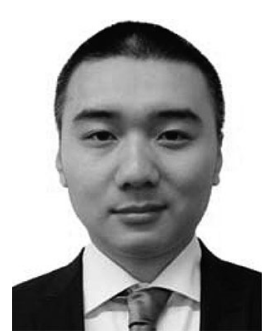

Yifei Shi (S'09-M' 12) was born in Jiangsu, China, in 1981. He received the B.E. degree in electronic and information engineering from Nanjing Tech University, Nanjing, Jiangsu, China, in 2004. He received the $\mathrm{Ph} . \mathrm{D}$. degree in electromagnetic field and microwave technology from Nanjing University of Science and Technology, Nanjing, Jiangsu, China, in 2012.

Since 2012, he has been a Postdoctoral Research Fellow with the Department of Electrical Engineering, King Abdullah University of Science and Technology, Thuwal, Saudi Arabia. His research interest includes time domain integral equation. 


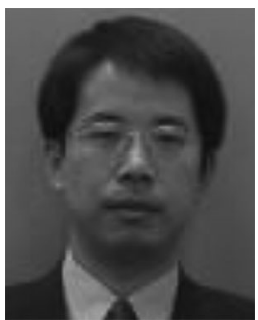

Li Jun Jiang (S'01-M'04-SM'13) received the B.S. degree in electrical engineering from the Beijing University of Aeronautics and Astronautics, Beijing, China, in 1993, the M.S. degree in electronic engineering from the Tsinghua University, Beijing, China, in 1996, and Ph.D. degree in electrical and computer engineering from the University of Illinois at UrbanaChampaign, Champaign, IL, USA, in 2004.

From 1996 to 1999, he was an Application Engineer with the Hewlett-Packard Company. Since 2004, he has been the Postdoctoral Researcher, the Research Staff Member, and the Senior Engineer at IBM T. J. Watson Research Center. Since the end of 2009, he has been an Associate Professor with the Department of Electrical and Electronic Engineering, University of Hong Kong, Hong Kong. He was the Scientific Consultant to Hong Kong ASTRI (Hong Kong Applied Science and Technology Research Institute Company Limited) in 2010-2011, the Panelist of the Expert Review Panel of Hong Kong R\&D Centre for Logistics and Supply Chain Management Enabling Technologies since January 1st, 2013. He is also the Senior Visiting Professor at Tsinghua University since June 2013.

Dr. Jiang was the TPC Chair of the 7th International Conference on Nanophotonics (ICNP)/the 3rd Conference on Advances in Optoelectronics and Micro/Nano Optics (AOM), the TPC Co-chair of the 12th International Workshop on Finite Elements for Microwave Engineering, the Co-chair of 2013 International Workshop on Pulsed Electromagnetic Field at Delft, the Netherlands, the General Chair of 2014 IEEE 14th HK AP/MTT Postgraduate Conference. He was the elected TPC Member of IEEE EPEP since 2014, the TPC Member of IEEE EDAPS since 2010, the TPC Member of 2013 IEEE ICMTCE, the Scientific Committee Member of 2010 IEEE SMEE, the Special Session Organizers of IEEE EDAPS, IEEE EMC, ACES, AP-RASC, PIERS, Co-organizer of HKU Computational Science and Engineering Workshops in 2010-2012, the TC-9 and TC-10 Member of IEEE EMC-S since 2011, and Session Chairs of many international conferences. He also was the Reviewer of the IEEE TRANSACTIONS ON SEVERAL TOPICS, and other primary electromagnetics and microwave related journals. He received the IEEE MTT Graduate Fellowship Award in 2003 and the Y. T. Lo Outstanding Research Award in 2004. He is the Associate Editor of the IEEE TRANSACTIONS ON ANTENNAS AND PROPAGATION, the Associate Editor of Progress in Electromagnetics Research, the Associate Guest Editor of the PROCEEDINGS OF IEEE SPECIAL ISSUE in 2011-2012, an IEEE AP-S Member, an IEEE MTT-S Member, an IEEE EMC-S Member, an ACES Member, and a Member of Chinese Computational Electromagnetics Society. He was the Semiconductor Research Cooperation (SRC) Industrial Liaison for several academic projects.

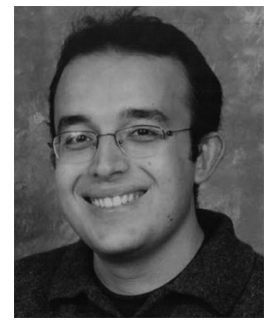

Hakan Bağicı (S'98-M'07-SM'14) received the B.S. degree in electrical and electronics engineering from the Bilkent University, Ankara, Turkey, in June 2001, and the M.S. and Ph.D. degrees in electrical and computer engineering from the University of Illinois at Urbana-Champaign (UIUC), Urbana, IL, USA, in August 2003 and January 2007, respectively.

From June 1999 to July 2001, he was an Undergraduate Researcher at the Computational Electromagnetics Group, Bilkent University. From August 2001 to December 2006, he was a Research Assistant at the Center for Computational Electromagnetics and Electromagnetics Laboratory, UIUC. From January 2007 to August 2009, he was a Research Fellow with the Radiation Laboratory, University of Michigan. In August 2009, he joined the Division of Physical Sciences and Engineering, King Abdullah University of Science and Technology as an Assistant Professor of electrical engineering. He authored and coauthored eight finalist papers in the student paper competitions at the 2005, 2008, and 2010 IEEE Antennas and Propagation Society International Symposiums and 2013 and 2014 Applied Computational Electromagnetics Society Conference. His research interests include various aspects of computational electromagnetics with emphasis on time-domain integral equations and their fast marching-on-in-time-based solutions, well-conditioned integral-equation formulations, and development of fast hybrid methods for analyzing statistical EMC/EMI phenomena on complex and fully loaded platforms.

Dr. Bağc1 received the 2008 International Union of Radio Scientists Young Scientist Award and the 2004-2005 Interdisciplinary Graduate Fellowship from the Computational Science and Engineering Department, UIUC. His paper titled "Fast and rigorous analysis of EMC/EMI phenomena on electrically large and complex structures loaded with coaxial cables" was one of the three finalist (with honorable mention) for the 2008 Richard B. Schulz Best Transactions Paper Award given by the IEEE Electromagnetic Compatibility Society. 